\title{
Vascular Fractality and Alimentation of Cancer
}

\author{
Andras Szasz \\ Department of Biotechnics, St. Istvan University, Budaors, Hungary \\ Email: biotech@gek.szie.hu
}

How to cite this paper: Szasz, A. (2021) Vascular Fractality and Alimentation of Cancer. International Journal of Clinical Medicine, 12, 279-296. https://doi.org/10.4236/ijcm.2021.127025

Received: June 3, 2021

Accepted: July 13, 2021

Published: July 16, 2021

Copyright (c) 2021 by author(s) and Scientific Research Publishing Inc. This work is licensed under the Creative Commons Attribution International License (CC BY 4.0).

http://creativecommons.org/licenses/by/4.0/ (c) (i) Open Access

\begin{abstract}
Background: The basal metabolic rate has a scaling by tumor mass on the exponent of 3/4, while a simple surface-supplied volume of the mass would have a lower exponent, $2 / 3$. The higher exponent can be explained by optimizing the overall energy distribution in the tumor, assuming that the target is four-dimensional. There are two possible ways of approximating the metabolic rate of the malignant tumor: 1) the volume blood-supply remains, but the surface and the length of the vessel network are modified; or 2) assuming that the malignant cell clusters try to maximize their metabolic rate to energize their proliferation by the longer length of the vessels. Our objective is to study how vascular fractality changes due to the greater demand for nutrients due to the proliferation of cancerous tissue. Results: It is shown that when a malignant tumor remains in expected four-dimensional volumetric conditions, it has a lower metabolic rate than the maximal metabolic potential in the actual demand of the proliferating cancer tissue. By maximizing the metabolic rate in malignant conditions, the allometric exponent will be smaller than $3 / 4$, so the observed "dimensionality" of the metabolic rate versus mass becomes greater than four. The first growing period is exponential and keeps the "four-dimensional volume", but the growth process turns to the sigmoidal phase in higher metabolic demand, and the tumor uses other optimizing strategies, further lowering the scaling exponent of metabolic rate. Conclusion: It is shown that a malignant cellular cluster changes its metabolic scaling exponent when maximizing its energy intake in various alimentary conditions.
\end{abstract}

\section{Keywords}

Allometry, Metabolism, Fractal Dimensions, Optimization, Cancer, Vascularity

\section{Introduction}

The highly organized living systems are energetically open and far from thermal 
equilibrium [1]. Its physical phenomena are collective and have strong physical roots [2]. Structures built up by anabolism and store information in the open system [3]. The living matter is heterogeneous, having numerous different electrolytes engulfed by specialized tissues or lipids enveloping isolated aqueous electrolytes in definite structures. The isolating layers control the chemical and physical reactions between the electrolytes and regulate the complex interactions. The fundamental division of electrolytes is between the cytosol (the intracellular electrolyte) and the Extracellular Matrix (ECM). The membrane is a complexly organized multifunctioning part. This double lipid layer regulates the information and ionic exchange between the intra and extracellular reagents, having a vital role in the energy distribution and production of the entire system. The mass of the living object is volume dependent (scaling by 3 ), while the surface is scaled only by 2 . Consequently, we expect an exponent for mass-dependence of energy exchange (metabolism) as $2 / 3$, the ratio of the cell surface to the cell volume $\left(\propto \frac{r^{2}}{r^{3}}=r^{2 / 3}\right)$. So the expected metabolic power $\left(P_{m e t}\right)$ in rest state (Basal Metabolic Power, $B M P)$ dependence vs. mass $(M)$ is expected:

$$
P_{\text {met }}=B M P \propto M^{\frac{2}{3}}=M^{\alpha}
$$

However, the experiments show a variation of exponents, the $\alpha=2 / 3$ is not shared. When the metabolism is concentrated on surfaces, the $\alpha \approx 2 / 3$ well approaches reality. On the other hand, when it is centered on the energy resources, the exponent is close to $\alpha \approx 3 / 4$. When the whole mass of the organism is involved in the metabolic energy exchange, the exponent is near to $\alpha \approx 1$. In complete demand, the actual body-part (organ or whole-body) needs maximally available energy supply, proportional to its mass, so the scaling exponent is $\alpha=1$ in this case [4]. In this case, the actual demand decides about the metabolic power and not the geometry. Of course, both the extremes are not ideal for the living object and could not follow evolutional requests. What is optimal? Despite the different exponential power, one feature is strictly common, all the experiments show power-scale (called scaling) in a few orders of magnitudes of the parameters, which is linear in the double logarithmic plot:

$$
\ln \left(P_{\text {met }}\right) \propto \alpha \cdot \ln (M)
$$

The scaling behavior is the consequence of the self-similarity of the living objects [5] [6]. The fundamental phenomenon behind it is the relative proportional change of the parameters [7]. The fundamental principle was oriented on the changes of the same organism, which has to grow in collective harmony, so the relative growth of parts must have balanced growth [8]. The structure and regulation of biosystems are complex. Various modern approaches have been developed in the last few decades to describe this complexity. The description of statistics of complex systems is far from the normal (Gaussian) distribution. Usually, power-law-tailed distributions (with a general exponent $\alpha$ ) are applied:

$$
f(x)=x^{\alpha}
$$


There are various phenomena, including social, economic, physical, chemical, and biological, to be described by this function [9] [10] [11] [12] [13]. Despite the somewhat different fields of applications of the power law, it has a common root in complex systems: self-organization. The simplest fingerprint of the self-organized complexity is the self-similar or scale-free structures characterized by a power function. This power-function relation magnifies the $f(x)$ by a constant only, $m$-dependent $\Xi=m^{\alpha}$ value at any $m$ magnification of $x$.

$$
f(m x)=(m x)^{\alpha}=m^{\alpha} x^{\alpha}=\Xi x^{\alpha}=\Xi f(x)
$$

Self-organization explains the evolution of the system [14], expressed in non-linear dynamics [15].

The objective of this present article focuses on analyzing the metabolic alimentation of the healthy tissues in normal conditions and the developing tumors in two different conditions:

1) When the tumor metabolizes as a homeostatic organized unit, the theoretically expected allometric exponent corresponds with the optimal healthy allometry;

2) When the tumor metabolism is not in such an "ideal" optimization of the metabolic supply, its alimentation is suboptimal, using the observed fractal behavior of its angio-structure.

\section{Method}

Fractal physiology describes the structural and dynamical properties of living organisms and their parts [16] [17], based on physical principles [18]. The self-similar behavior could be described by the normalized relative change of the magnitudes, similarly to the Weber-Fechner law [19] in psychophysics like:

$$
\frac{\Delta f}{f}=\alpha \frac{\Delta x}{x}
$$

where $\alpha$ is a constant fitting factor. By integration, we get the (3):

$$
\ln (f)=\alpha \ln (x) \rightarrow f(x)=x^{\alpha}
$$

The self-similar functional relation makes a "scale-invariance" feature due to the independence of the magnification, which is the fundamental behavior of the fractal structures, too [20].

The power function is the central description of the scaling in (1), which bases the allometry of living organisms [21]. The original allometry idea was recognized almost a hundred years ago [22], but the exciting question of the energizing of the life phenomena explained in connection of allometry is a half-century-old knowledge [23]. The connection between the homeostatic energizing level and the basal metabolic rate $\left(B_{0}\right)$ as a self-similar function of mass $(m)$ of living objects is [24]:

$$
B_{0}=a m^{\alpha}
$$


where the two parameters are determined experimentally; $a$ is the allometric coefficient, and $\alpha$ is the allometric exponent, and (7) is usually called bioscaling [25]. The usual regression analysis uses the logarithmic transformation of (7):

$$
\ln B_{0}=\alpha \ln (m)+\ln (a)
$$

which allows high linear accuracy and fits both parameters $a$ and $\alpha$ well [26]. The literature has numerous debates about the theoretical allometric relation based on fractal calculus and the empirical fits based on probability calculus [27]. The $B_{0}$ of living objects shows allometric scaling to its mass, which refers to the energy supply of the living mass of the volume. The (7) function gives a correct mathematical and biological framework for the complex bio-systems fractal studies [28]. The scaling power function of the mass describes it, and it has been shown valid in a broad category of living structures and processes [29]. The scaling considerations are applied not only in biology but broader, in the complete biosystem as well [30]. The importance of understanding the challenges of the complexity of human medicine was recognized on this basis [31] [32].

In a simple formulation, metabolic processes are surface-dependent, while the mass is proportional to the volume. Therefore, the exponent of their ratio mirrors their dimensionality, and consequently, the exponent is $2 / 3$ [33]. Complex living allometry most likely shows the exponent as $3 / 4$ instead of $2 / 3$ in a broad spectrum of living objects [34], or at least have no linearity in a double-logarithmic plot [35]. However, the large data-mining does not show an overall validity of the $3 / 4$ exponent over $2 / 3$ [36]. The curvature could be size-dependent in developing clusters by their size [37]. The $3 / 4$ exponent could be described as a relation between the three-dimensional surface and the four-dimensional volume [38]. The explanation of the fourth dimension is based on the fractal structure of microcirculation [39], which supplies the energy demand according to a homeostatic equilibrium $\left(B_{0}\right)$ in the living complexity. Life in this meaning is "four-dimensional". Its metabolic exchange processes proceed on fractal surfaces, maximizing the available energy consumption, scaling even the fluctuation of the metabolic power in the universal scaling law as well [40].

The optimization of energy consumption was formulated rigorously by the scaling idea and discussed in a universal frame, even on the energy-consumption subcellular level, including the mitochondria and respiratory complexes [41]. The allometry shows a structural, geometrical constraint for living organisms in homeostatic equilibrium.

The metabolic scaling in cancer development is critical [42]. Contrary to the homeostatic homogeneity of the healthy tissue [43]; the functional heterogeneity of the solid tumor allows an abnormal organ self-possession of multiple cell-types and electrolytes like the Extracellular Matrix (ECM) lymph and blood-transports [44]. The tumor metabolism is based on the blood transport to the tumor. The logarithm of wet-weight of the tumor $\left(m_{\text {wet }}\right)$ and the tumor blood-flow $\left(B_{t}\right)$ have linear dependence [45], which was observed in model xenografts of ovarian cancer, so they have a bioscaling relation: 


$$
\begin{aligned}
& \log \left(B_{t}\right)=-0.808 \log \left(m_{\text {wet }}\right)-0.436, r^{2}=0.79, p<0.001 \\
& {\left[B_{t}=0.6466 \cdot m_{\text {wet }}^{-0.808}\right]}
\end{aligned}
$$

where the exponent is close to $3 / 4$.

The allometric scaling supposes three geometrical variables to define the optimization of the circulatory system in living objects:

- The average length of the blood circulatory network (I);

- The surface of the relevant material exchange of the blood circulation system $(s)$;

- The volume of the blood ( $v)$.

Furthermore, we suppose that these parameters are represented by the self-similar, self-organized functions of the $L$ value, which is characteristic of a given organ. Hence:

$$
l \propto L^{a_{l}}, s \propto L^{a_{s}}, v \propto L^{a_{v}}
$$

Using the theoretical fractal explanation, the conditions are: $a_{l} \geq 1, a_{s} \geq 2$ and $a_{v} \geq 3$, from where:

$$
l \propto L_{0}^{1+\varepsilon_{l}}, s \propto L_{0}^{2+\varepsilon_{s}}, v \propto L_{0}^{3+\varepsilon_{v}}
$$

where $0 \leq \varepsilon_{l} \leq 1,0 \leq \varepsilon_{s} \leq 1$, and $L_{0}$ is the characteristic length. The first relation limits the pattern of the circulatory system to the maximum that could be planar, while the second is limited to a maximum, filling up a three-dimensional space. The third exponent $\varepsilon_{v}$ could be calculated because the exponents are not independent. The volume is proportional to the product of the surface and length:

$$
v \propto s \times l
$$

consequently

$$
\varepsilon_{v}=\varepsilon_{l}+\varepsilon_{s}
$$

Using these conditions, we obtain from (11):

$$
L_{0} \propto v^{\frac{1}{3+\varepsilon_{v}}} \rightarrow s \propto v^{\frac{2+\varepsilon_{s}}{3+\varepsilon_{v}}}
$$

Furthermore, the actual volume of the blood is proportional to the actual mass of the given system or organ:

$$
v \propto L_{0}^{3+\varepsilon_{v}} \propto m^{1}
$$

Considering (14) and (15), now we have:

$$
s \propto m^{\frac{2+\varepsilon_{s}}{3+\varepsilon_{l}+\varepsilon_{s}}}
$$

The metabolism is a surface-controlled mechanism, so $(B M R) \propto s$, consequently:

$$
B_{0} \propto m^{\frac{2+\varepsilon_{s}}{3+\varepsilon_{l}+\varepsilon_{s}}}
$$

If the living structure is geometric in conventional Euclidean meaning, then 
$\varepsilon_{l}=\varepsilon_{s}=\varepsilon_{v}=0$ and therefore $a_{l}=a_{s}=a_{v}$; consequently, the scaling is $(B M R) \propto m^{\alpha}$, where $\alpha=\frac{2+\varepsilon_{s}}{3+\varepsilon_{l}+\varepsilon_{s}}=2 / 3$. When at least one of the $\varepsilon_{l} \neq 0$, $\alpha \neq 2 / 3$, which modifies the common simple dimensional approach of the metabolic processes.

\section{Results}

The allometry gives a possibility to describe the development of the tumor [46]. It is valid for the primary cancer lesions but not always applicable in metastases [47]. We are dealing with primary tumors only. There are two ways of approximating the allometric metabolic rate of a tumor:

1) The theoretical approach accepts that a healthy life has a four-dimensional behavior connected to the highly self-organized, consequently self-similar hierarchic order [48], we fix the exponent to $3 / 4$.

2) The experimental approach assumes that the cell cluster tries to maximize its metabolic rate [49], and this way, it modifies the scaling exponent from the value of $3 / 4$.

Both approaches depend on the environmental conditions of the tumor, mainly on the nourishment of the cells.

\subsection{Optimal Alimentation to Maximum Metabolic Rate}

Evolution maximized the surface where the nutrients are transferred from the blood to the cells, ensuring the best conditions of the living object, so:

$$
s\left(\varepsilon_{l}, \varepsilon_{s}\right)=\max
$$

This task is equivalent to the minimizing of the reciprocal value of the exponent in (16):

$$
\frac{3+\varepsilon_{l}+\varepsilon_{s}}{2+\varepsilon_{s}}=\min
$$

with constraint conditions of:

$$
0 \leq \varepsilon_{l} \leq 1,0 \leq \varepsilon_{s} \leq 1
$$

(19) can be transformed into

$$
\frac{3+\varepsilon_{l}+\varepsilon_{s}}{2+\varepsilon_{s}}=1+\frac{1+\varepsilon_{l}}{2+\varepsilon_{s}}=\min
$$

Hence, considering (20), the minimum condition demands that:

$$
\varepsilon_{l}=0, \varepsilon_{s}=1
$$

Substituting (22) into (11), the exponents of the self-similar structures are:

$$
l \propto L_{0}^{1}, \quad s \propto L_{0}^{3}, \quad v \propto L_{0}^{4}
$$

Consequently, in cases of ideal alimentation, these exponents are the fractal dimensions of the parameters of the network, and while the length is one dimensional, the surface is three, and the volume is four [38]. Because metabolism 
is a surface-regulated process, $s \propto(B M R)$, the scaling exponent of the metabolic rate versus mass using (16) is $3 / 4$ :

$$
B_{0} \propto S \propto m^{\frac{2+\varepsilon_{s}}{3+\varepsilon_{l}+\varepsilon_{s}}}=m^{\frac{3}{4}}
$$

and so $B_{0}$ in the unit mass

$$
\frac{B_{0}}{m} \propto m^{-\frac{1}{4}}
$$

Primarily the blood stream provides the metabolic supply, so the fractality of the vascular network could be decisional in its allometric evaluation. The condition of (24) maximizes the blood flow energizing all the parts of the volume for their optimum, providing a maximum metabolic rate.

In consequence of (24), the life prefers the large masses as more effective energy-consumers in a unit volume shown in (25). However, on another side this process could lead to the loss of complex information, developing higher instability of the system, arguing that this is a negative tendency manifest the "aging of life's algorithm as a whole" [50]. The model could be applied by guessing when the energy supply is optimal, so the developed active surface cannot supply the actual demands. Two different sources are possible to create such a situation (1) the length of the supplier system changes (the constructional template differs), or (2) the volume of transport exchange is limited despite the growing demands. Various irregularities originate both challenges could be a symptom of disease, like cancer [51].

\subsection{Suboptimal Alimentation for Tumor}

The malignancy usually demands a higher energy input from its healthy environment than the available. The tumor supply is suboptimal. The higher energy demand (usually exponential in starting phase [52]) forces to increase the length of the vessel network. In cancerous clusters, contrary to (22), the vascular fractal dimension $\left(D_{v}\right)$ of the supplying blood-vessel network $\left(l \propto L_{0}^{D_{v}}\right)$ is larger than 1 , $\left(D_{v} \geq 1\right)$ [51]; consequently $\varepsilon_{l} \neq 0$ in the relation of (11). $D_{v}$ could be measured by the box-counting method [51].

According to (11), the actual active surface is evolutionary normal for self-organizing of healthy tissues ( $s \propto L_{0}^{3}$ ). The extra energy demand of the intensive proliferation changes the exponents of parameters in (11). In this case, the surface of the supply follows the evolution-requested exponent of $3\left(\varepsilon_{s}=1\right.$ from (22)) in the self-similar conditions, but the requested length changes:

$$
l \propto L_{0}^{1+\varepsilon_{l}}=L_{0}^{D_{v}}, s \propto L_{0}^{3}
$$

where $\varepsilon_{l}>0$ modifies the power of the transport measures, so the fractal organization of the transport lines is different. This type of change could be formed by neoangiogenesis satisfying the higher energy demand in cancerous tissues and could cause abnormalities as inflammation, thrombosis, varicose veins modification of the arteries, etc. The corresponding power-law for the ac- 
tual metabolic rate at the longer length of vessels, so the suboptimal metabolic rate in this phase $\left(B_{s o 1}\right)$ from (24) is:

$$
B_{\text {sol }} \propto m^{\frac{2+\varepsilon_{s}}{3+\varepsilon_{l}+\varepsilon_{s}}}=m^{\frac{3}{4+\varepsilon_{l}}}=m^{\frac{3}{3+D_{v}}}
$$

The apparent "dimension" of the reaction request for volume is $\left(4+\varepsilon_{l}\right)>4$, the dimension increases. According to $l \propto L_{0}^{1+\varepsilon_{l}}$ the measurable fractal dimension of the blood vessel network is $D_{v}=1+\varepsilon_{l}$. In this way the $\varepsilon_{l}$ is measurable by the fractal dimension of the vessel structures [53], for example, with the box-counting method [51]. When $D_{v}=1.3$ [53], $\varepsilon_{l}=0.3$, and the scaling exponent is $\alpha \cong 0.7>2 / 3$. The Microvessel Fractal Dimension (MFD) (which is equivalent with $\left(1+\varepsilon_{l}\right)$ for renal cell carcinoma ranges between $1.30-1.66$ [54], and correlates well with the tumor Microvessel Density ( $M V D$ ) [54]. From (27) we know, when $\varepsilon_{l}=0.478$, the scaling exponent describes a non-fractal-like structure, $\alpha=2 / 3$.

When the tumor growth is so intensive that the available length of the vessel network cannot deliver appropriate energy, then another possible deviation from the homeostatic self-organization happens. In this case, the volume of the delivered energy remains constant, which limits the energy supply. The tumor-growth turns to sigmoidal this stage [55], usually follows Weibull distribution due to the self-similar development [6]. This could happen in severe hypoxia, low oxygen saturation in blood, anemia, various hematological diseases. In this case, the volume of the supply follows the evolution-requested exponent of $4\left(\varepsilon_{v}=1\right)(23)$, in the self-similar conditions in [53], but the requested length and surface is not enough for the proper work, so $\varepsilon_{l}^{\prime}>1$ and $\varepsilon_{s}<1$. The self-similar conditions differ from (22) due to (13):

$$
l \propto L_{0}^{1+\varepsilon_{i}^{\prime}}, s \propto L_{0}^{3-\varepsilon_{i}^{\prime}}, v \propto L_{0}^{4}
$$

Consequently, at fixed four-dimensional volume, the metabolic surface reactions behave by power-law of suboptimal metabolic rate in this phase:

$$
B_{s o 2} \propto m^{\frac{2+\varepsilon_{s}}{3+\varepsilon_{i}^{\prime}+\varepsilon_{s}}}=m^{\frac{3-\varepsilon_{1}^{\prime}}{4}}=m^{\frac{4-D_{v}^{\prime}}{4}}
$$

Here the volume "dimension" of the reaction request is 4 , but the actual conditions are worse than optimal. The $\varepsilon_{l}^{\prime}$ again here also is measurable by the fractal dimension of the structures [56], in this case, the fractal dimension of the vessel system is $D_{v}^{\prime}=1+\varepsilon_{l}^{\prime}$. For example, measuring the vascular fractal dimension in one disease as $D_{v}^{\prime}=1.41$ [51], we use $\varepsilon_{l}^{\prime}=0.41$, so the scaling exponent is $\alpha=0.65$. When $\varepsilon_{l}^{\prime}=0.28$ [56], the scaling exponent is $p=0.68$. At $\varepsilon_{l}^{\prime}=0.33$, the scaling exponent is the well-known $\alpha=2 / 3$.

The exponents of the active transport surface in the two suboptimal supplies

$$
\alpha=\frac{3}{4+\varepsilon_{l}}=\frac{3}{3+D_{v}} \text { and } \alpha^{\prime}=\frac{3-\varepsilon_{l}^{\prime}}{4}=\frac{4-D_{v}^{\prime}}{4}
$$

Both these exponents are smaller than the optimal, and the exponent in the second phase of growth is the smallest (Figure 1). 


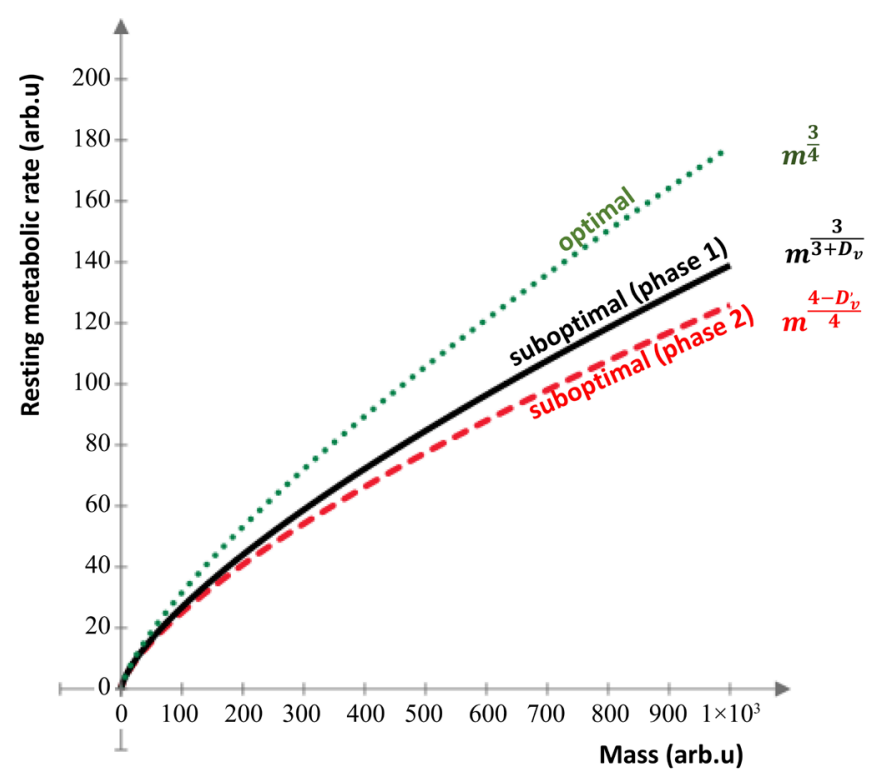

Figure 1. The allometric development with the various exponents. The initial phase of tumor growth is exponential, and the allometric scale follows the phasel curve, while the intensive development further decreases the exponent, which turns to a sigmoid phase in a tumor-specific time.

Following the idea of "4-dimensionality", the volume dimension changes in $\alpha$, while the active surface in $\alpha^{\prime}$. Both exponents are $\leq 3 / 4$ because of the length fractal dimension $>1$. The mechanisms which cause this modification are different. The same exponent could be only in Euclidean non-fractal case, when $D_{v}=D_{v}^{\prime}=1$. Due to $D_{v}<3$ and $D_{v}^{\prime}<3$, hence $\alpha>0.5$ and $\alpha^{\prime}>0.25$. The Mandelbrot calculated 2.7 for the fractal dimension of the arterial tree of the lung [57], which was supported by experiments later [58], the relevant changes are $\alpha \approx 0.526$ and $\alpha^{\prime} \approx 0.325$. Both values are smaller than $\alpha=2 / 3$.

Both non-optimal situations (defect of the length of transport way or limited transport against the demands) make the tissue under-energized, and the exponent of the power-relation scaling down-regulated. In such a way, measuring the scaling exponent of metabolism and the fractal dimension of the supplying microvessels have a diagnostic value about the actual deviations from normal.

\section{Discussion}

The optimal alimentation in a healthy system makes the energy distribution balanced, supplying all requirements of the homeostatic state. The exponent $3 / 4$ has a strong predominance on a theoretical and empirical basis [59] in healthy homeostatic basal metabolic activity. The ideal nutrition supply supports ontogenic growth. However, at least at larger sizes, the cancer growth never happens with an optimal nutrition supply; the cells compete intensively for the available energy sources.

The cancer is out of the overall homeostatic balance. The tumor development certainly has a higher energy supply due to its proliferation than its healthy 
counterpart needs. Due to the extra-large energy demand, the tumor development's alimentation in most cases is far from optimal, so the tumor is in a permanent energy deficiency. When the oxygen supply is limited, the first attempt to produce more ATP is the massive fermentative use of glucose, a simple and quick production mechanism. The cell extends its ATP production to fermentation by non- mitochondrial respiration, abandoning the more complicated Kerbs-cycle in the mitochondria [60].

While the mitochondrial metabolism is always aerobic, its scaling exponent is nearly $\alpha=3 / 4$ [30]. However, the scaling of metabolic activity is also different in mitochondrial and non-mitochondrial processes [61]. The metabolic power not only depends on the active surface of the transport but also on the transport rate at the same active surface size. Due to the transport modifications at the changed metabolic pathway, the deviation from the $3 / 4$ exponent could be remarkable. The allometric scaling exponent of fermentative processes decreases to nearly $2 / 3$. This last scaling exponent shows that the cell-membrane directly regulates the fermentation, and the surface/volume ratio controls the complete process, which could be anticipated from the direct linear dependence of the lactate production $\left(V_{L}\right)$ on the glucose-intake $\left(V_{G}\right)$ with a slope of $\cong 1$ [45]:

$$
\log \left(V_{L}\right)=+0.977 \log \left(V_{G}\right)+0.108, r^{2}=0.72, p<0.001
$$

while the bioscaling of the oxygen $\left(V_{\mathrm{O}_{2}}\right)$ and glucose $\left(V_{G}\right)$ intake [45] are even lower than $2 / 3,-0.570$, and -0.523 , respectively.

Not only does the malignancy need an intensive extra metabolism. For example, the benthic invertebrates $(n=215)$ have the lowest average scaling exponent $\left(\alpha_{\text {mean }}=0.63\right.$, [near to 2/3], $\left.C I_{\text {mean }}=0.18\right)$, which metabolizes in an anaerobic way [62]. No regulative factor exists when the cells are entirely independent, and the available alimentation is unlimited (like in most in vitro experiments). The metabolic rate is linearly proportional to the mass, so the exponent is $\alpha \cong 1$ [30].

The metabolic transformation of the cells [63] is one of the well-recognized hallmarks of malignancy [64] that has an emerging intensive interest in the field of oncology [65], as the core hallmark of cancer [66]. The adaptation of mitochondria in energy-limited conditions is the focus of the research [67]. The tumor forces the development of the angiogenetic processes [68] and overcomes the energy limitations. The vascularity is promoted [69], and the rapid development by intensive proliferation supports the changes of the scaling behavior [70]. Without extra angiogenesis (starting clusters), only the ready-made capacity of the delivery is available, so the tumor has a suboptimal alimentation. In the beginning, its fractal structure was developed, which is similar to the healthy structure, so the four-dimensional scaling remains valid (28).

When the tumor develops, the fractal structure of vascularity changes. Consequently, its fractal dimension changes too. The forced angiogenesis [68] tries to provide a sufficient supply to the hypoxic (insufficiently supported) tumor, and the structure changes rapidly, broadening the scaling exponent in a wide range [71]. The missing supply suppresses the scaling exponent, shown in (27). 
Still, the angiogenetic pool changes the trend, approaching linearity. The unlimited availability of nutrients for every cell realizes the linearity measured in vitro [48], limited to $\sim 0.9$ in vivo by insufficient oxygen transport [72]. However, the angiogenesis is usually not fast enough to supply the faster-growing larger tumors, so the inner part of the tumor becomes necrotic, forming a smaller living mass to supply, easing the energy distribution [68]. The essential message of the cases of insufficient alimentation from the calculations above is that when the fractal dimension of the supplying network grows, the scaling exponent decreases. The four-dimensionality and the allometry with the evolutional optimizing request are not the same approaches: further evolution conditions have a higher than four-dimensional allometric scaling. The tumor mass is a somewhat indefinite parameter because the whole environment of the tumor suffers from suboptimal alimentation. Consequently, we tried to find a more fundamental networking condition parameter published elsewhere [73].

There is a vast number of researches about the vascular development of the tumor progression, calculating the fractal dimension of the vascularity. The in silico modeling of the growing tumor vessel architecture in high-grade gliomas [74] shows that the fractal dimension is less than 1 in the avascular state and growing linearly by time, reaching $D_{t=2760 \mathrm{~h}} \cong 1.2$ at $t=2760 \mathrm{~h}$, by slope approximately $\cong 6.2 \times 10^{-4}$. In a longer time, the development of the fractal dimension drastically changes, follows a less rapid development (slope $\cong 2.5 \times 10^{-4}$ ) until $D_{t=4000 \mathrm{~h}} \cong 1.48$. We may assume that the fractal dimension 1.2 characterizes the finally developed vessel structure inside the tumor, followed by neo-angiogenetic processes reaching the tumor-surface, changing the vascular architecture, growing slower to the higher values of the fractal dimension.

In optimal alimentation, the allometric scaling shows exponent 3/4 (24); which supposes the $l \propto L_{0}^{1}$, so the vascular fractal dimension in this case is $D_{v a}=1$. However $D_{v a}>1$ by the growing vessel network, so $\alpha<3 / 4$ in the allometric scaling of tumor-vascularity due to the suboptimal energy supply, which triggers the angiogenesis. Using the results from in silico model-calculations, the internal growth of the vessels have $\left(B_{0}\right)_{i 1} \propto m^{\frac{2.8}{4}}=m^{0.700}$, or $\left(B_{0}\right)_{i 2} \propto m^{\frac{3}{4.2}}=m^{0.714}$, according to the assumption of suboptimal alimentation by maximal metabolic rate (case 1) or by the metabolic rate forced four-dimensional "optimizing" concept (case 2). When the external angiogenesis is developing, the allometry changes: $\left(B_{0}\right)_{e 1} \propto m^{\frac{2.52}{4}}=m^{0.63}$, and $\left(B_{0}\right)_{e 2} \propto m^{\frac{3}{4.48}}=m^{0.67}$. So, the optimizing of the suboptimal energy availability in extended angiogenetic cases realizes the allometry, which fits the simple geometrical expectations $\alpha=2 / 3$ well.

The measurements of vascular fractal dimensions in various tumors show a lower scaling exponent than the ideal $3 / 4$, depending on the conditions of the tumor-angiogenesis development. For example, when the epithelial-connective tissue interfaces with a malignant tumor in the oral mucosa, it is $D_{v a} \geq 1.41$ [75], the scaling exponent in suboptimal alimentation situations in cases 1 and 2 
are $\alpha \cong 0.64$ and $\alpha=0.68$. The last one (optimal distribution of the suboptimally available energy) is near the "conventional" $2 / 3$. Another microscopic evaluation of angio-structures [76] shows lower values of $\alpha$, like the fractal dimension of the normal and malignant tissues are $D_{\text {healthy }} \cong 1.65$ and $D_{\text {malignant }} \cong 1.74$, respectively [77]; resulting in low $\alpha$ values. In other evaluations, the vascular structure's dimensionality grows to 1.9 , which provides the maximal energy usage of the suboptimal alimentation, and the exponent became as low as $\alpha=0.525$.

It is interesting to see the effect of various anti-tumor treatments on the vascular fractal dimension. The treatment changes the vascularization and suppresses the fractal dimension forms $1.1351 .037,0.933,0.982$ by Photodynamic Therapy (PDT); Cysteine Proteases Inhibitors (CPI), combined therapy, PDT and CPI [78]; which corresponds in cases when the maximalizing of the energy-supply is equivalent to the allometric exponents of $0.716,0.741,0.767$ and 0.755 , respectively (the optimal distribution of the suboptimal availability would be $0.726,0.743,0.763$, and 0.753 ). By treating VEGF165, the fractal dimension increases from 1.65 to 1.69 , decreasing the allometric exponent [79] [80]. In matrigel inoculated human umbilical vein endothelial cells (HUVEC) treated by docetaxel, the fractal dimension of the vascular structure has decreased from 1.2 to 1.09 , corresponding in case $1 \alpha_{\text {control }} \cong 0.70 ; \alpha_{\text {treated }} \cong 0.73$, and in case 2 $\alpha_{\text {control }} \cong 0.71 ; \alpha_{\text {treated }} \cong 0.73$ [81]. The fractal analysis is a successful and rather accurate method for monitoring the efficacy of angiogenic consequences of therapies [82].

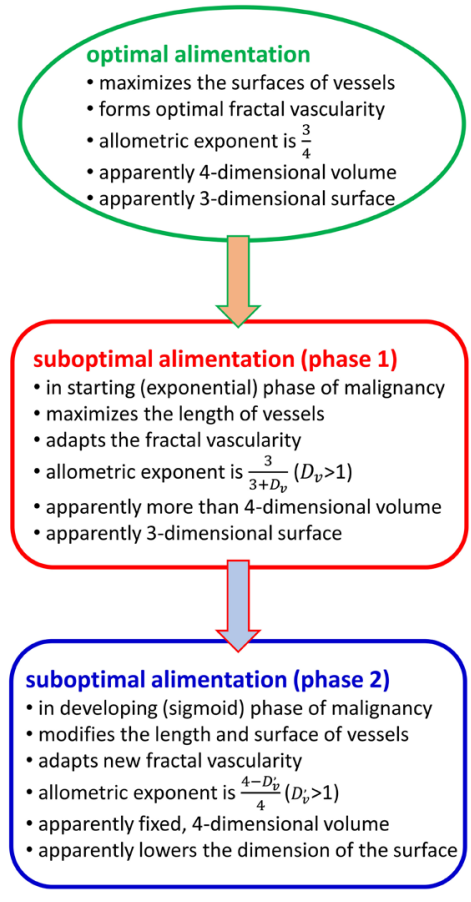

(a)

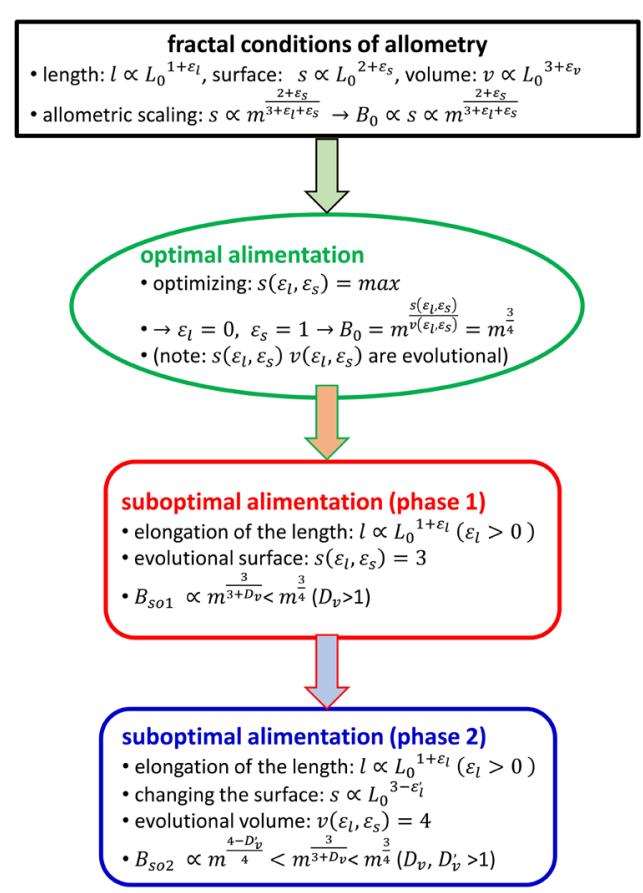

(b)

Figure 2. The summary of the structure of calculation. (a) The biophysical considerations (b) The mathematical description. 


\section{Summary}

We had shown that the allometric relation of basal metabolic rate and the tumor mass depends on the fractal dimension of the vascular structure. Due to the desperate need for energy supply and the intensive proliferation of the malignant tumor, cancer does not have an optimal alimentation. Two strategies could distribute the available (not sufficient) energy by the main transport of it, the vascular network:

1) Assuming that the cell cluster tries to maximize its metabolic rate by the surface transports and lowers the scaling exponent from the value of $3 / 4$;

2) Accepting that in the case of a four-dimensional volumetric behavior limits the energy supply. The tumor optimizes the energy distribution in its volume in among these conditions.

The structure of the biophysical considerations and their mathematical steps are summarized in Figure 2.

The two strategies in consequent phases of tumor growth optimize the available energy by different allometric scalings. The organized optimum of the suboptimal availability of energy gives lowered allometric scaling exponents.

\section{Acknowledgements}

This work was supported by the Hungarian National Research Development and Innovation Office PIACI KFI grant: 2019-1.1.1-PIACI-KFI-2019-00011.

\section{Conflicts of Interest}

The author declares no conflicts of interest regarding the publication of this paper.

\section{References}

[1] Barge, L.M., Branscomb, E., Brucato, J.R., Cardoso, S.S.S., Cartwright, J.H.E., Danielache, S.O., et al. (2017) Thermodynamics, Disequilibrium, Evolution: Far-FromEquilibrium Geological and Chemical Considerations for Origin-Of-Life Research. Origins of Life and Evolution of the Biospheres. The journal of the International Society for the Study of the Origin of Life, 47, 39-56. https://doi.org/10.1007/s11084-016-9508-Z

[2] Goldenfeld, N. and Woese, C. (2011) Life Is Physics: Evolution as a Collective Phenomenon Far from Equilibrium. Annual Review of Condensed Matter Physics, 2, 375-399. https://doi.org/10.1146/annurev-conmatphys-062910-140509

[3] Schrodinger, E. (1944) What Is Life? The Physical Aspect of the Living Cell. Cambridge University Press, Cambridge.

[4] Glazier, D.S. (2008) Effects of Metabolic Level on the Body Size Scaling of Metabolic Rate in Birds and Mammals. Proceedings of the Royal Society B: Biological Sciences, 275, 1405-1410. https://doi.org/10.1098/rspb.2008.0118

[5] Szasz, O., Szigeti, G.P. and Szasz, A. (2017) On the Self-Similarity in Biologyical Processes. Open Journal of Biophysics, 7, 183-196.

https://doi.org/10.4236/ojbiphy.2017.74014

[6] Szasz, O. and Szasz, A. (2020) Parametrization of Survival Measures, Part I: Conse- 
quences of Self-Organizing. International Journal of Clinical Medicine, 11, 316-347. https://doi.org/10.4236/ijcm.2020.115031

[7] Huxley, J.S. and Teissier, G. (1936) Terminology of Relative Growth. Nature, 137, 780-781. https://doi.org/10.1038/137780b0

[8] Huxley, J.S. (1932) Problems of Relative Growth. Book Review by C.H.K. in Gahan: New Parasitic Ilymenoplera, Lincoln Mac Veagh_The Dial Press, New York, 757.

[9] Newman, M.E.J. (2005) Power Laws, Pareto Distributions and Zipf s Law. Contemporary Physics, 46, 323-351. https://doi.org/10.1080/00107510500052444

[10] Mitzenmacher, M. (2004) A Brief History of Generative Models for Power Law and Lognormal Distributions. Internet Mathematics, 1, 226-251. https://doi.org/10.1080/15427951.2004.10129088

[11] Fisher, M.E. (1998) Renormalization Group Theory: Its Basis and Formulation in Statistical Physics. Reviews of Modern Physics, 70, 653-681. https://doi.org/10.1103/RevModPhys.70.653

[12] Barabasi, A. and Albert, R. (1999) Emergence of Scaling in Random Networks. Science, 286, 509-512. https://doi.org/10.1126/science.286.5439.509

[13] Chignola, R., Sega, M., Stella, S., Vyshemirsky, V. and Milotti, E. (2014) From Single-Cell Dynamics to Scaling Laws in Oncology. Biophysical Reviews and Letters, 9 , 273-284. https://doi.org/10.1142/S1793048014300035

[14] Kauffman, S.A. (1992) The Origins of Order: Self-Organization and Selection. In: Varela, F.J. and Dupuy, J.P., Eds., Understanding Origin, Vol. 130, Springer, Dordrecht, 153-181. https://doi.org/10.1007/978-94-015-8054-0 8

[15] Walleczek, J. (Ed.) (2000) Self-Organized Biological Dynamics \& Nonlinear Control. Cambridge Univesity Press, Cambridge. https://doi.org/10.1017/CBO9780511535338

[16] Bassingthwaighte, J.B., Leibovitch, L.S. and West, B.J. (1994) Fractal Physiology. Springer, New York. https://doi.org/10.1007/978-1-4614-7572-9

[17] Deering, W. and West, B.J. (1992) Fractal physiology. IEEE Engineering in Medicine and Biology, 11, 40-46. https://doi.org/10.1109/51.139035

[18] Musha, T. and Sawada, Y., Eds. (1994) Physics of the Living State. IOS Press, Amsterdam.

[19] Fechner, G.T., Howes, D.H. and Boring, E.G., Eds. (1966) Elements of Psychophysics. Volume 1, Adler, H.E., Trans., Holt, Rinehart and Winston, New York.

[20] Mandelbrot, B.B. (1967) How Long Is the Coast of Britain? Statistical Self-Similarity and Fractional Dimension. Science, 156, 636-638.

https://doi.org/10.1126/science.156.3775.636

[21] West, B.J. and West, D. (2012) Fractional Dynamics of Allometry. Fractional Calculus \& Applied Analysis, 15, 70-96. https://doi.org/10.2478/s13540-012-0006-3

[22] Huxley, J.S. (1932) Problems of Relative Growth. Johns Hopkins University Press, Methuen, London, 273.

[23] Kleiber, M. (1961) The Fire of Life: An Introduction to Animal Energetics. Wiley, New York.

[24] Savage, Van M., Allen, A.P., Brown, J.H., Gillooly, J.F., Herman, A.B., Woodruff, W.H. and West, G.B. (2007) Scaling of Number, Size, and Metabolic Rate of Cells with Body Size in Mammals. Proceedings of the National Academy of Sciences of the United States of America, 104, 4718-4723.

https://doi.org/10.1073/pnas.0611235104 
[25] Brown, J.H. and West, G.B., Eds. (2000) Scaling in Biology. Oxford University Press, Oxford.

[26] Mascaro, J., Litton, C.M., Hughes, R.F., Uowolo, A. and Schnitzer, S.A. (2014) Is Logarithmic Transformation Necessary in Allometry? Ten, One-Hundred, OneThousand-Times Yes. Biological Journal of the Linnean Society, 111, 230-233. https://doi.org/10.1111/bij.12177

[27] West, D. and West, B.J. (2012) On Allometry Relations. International Journal of Modern Physics B, 26, Article ID: 1230010.

https://doi.org/10.1142/S0217979212300101

[28] Brown, J.H., West, G.B. and Enquis, B.J. (2005) Yes, West, Brown and Enquist's Model of Allometric Scaling Is Both Mathematically Correct and Biologically Relevant. Functional Ecology, 19, 735-738.

https://doi.org/10.1142/S0217979212300101

[29] Calder III, W.A. (1984) Size, Function and Life History. Dover Publications Inc., Mineola, New York.

[30] West, G.B. and Brown, J.H. (2005) The Origin of Allometric Scaling Laws in Biology from Genomes to Ecosystems: Towards a Quantitative Unifying Theory of Biological Structure and Organization. Journal of Experimental Biology, 208, 1575-1592. https://doi.org/10.1242/jeb.01589

[31] West, B.J. (2006) Where Medicine Went Wrong: Rediscovering the Path to Complexity. Vol. 11, World Scientific Publishing Co. Pte. Ltd., New Jersey, London. https://doi.org/10.1142/6175

[32] West, B.J. (1990) Fractal Physiology and Chaos in Medicine. World Scientific, Singapore, London.

[33] White, C.R. and Seymour, R.S. (2003) Mammalian Basal Metabolic Rate Is Proportional to Body Mass2/3. Proceedings of the National Academy of Sciences of the United States of America, 100, 4046-4049. https://doi.org/10.1073/pnas.0436428100

[34] Moses, M.E., Hou, C., Woodruff, W.H., West, G.B., Nekola, J.C., Wenyun Zuo, et al. (2008) Revisiting a Model of Ontogenic Growth: Estimating Model Parameters from Theory and Data. American Naturalist, 171, 632-645. https://doi.org/10.1086/587073

[35] Kolokotrones, T., Savage, Van M., Deeds, E.J. and Fontana, W. (2010) Curvature in Metabolic Scaling. Nature, 464, 753-755. https://doi.org/10.1086/587073

[36] Dodds, P.S., Rothman, D.H. and Weitz, J.S. (2001) Re-Examination of the "3/4-Law" of Metabolism. Journal of Theoretical Biology, 209, 9-27.

https://doi.org/10.1006/jtbi.2000.2238

[37] Milotti, E., Vyshemirsky, V., Stella, S., Dogo, F. and Chignola, R. (2017) Analysis of the Fluctuations of the Tumour/Host Interface. Physica A. Statistical Mechanics and its Applications, 486, 587-594. https://doi.org/10.1016/j.physa.2017.06.005

[38] West, G.B., Brown, J.H. and Enquist, B.J. (1999) The Four Dimension of Life: Fractal Geometry and Allometric Scaling of Organisms. Science, 284, 1677-1679. https://doi.org/10.1126/science.284.5420.1677

[39] West, G.B., Brown, J.H. and Enquist, B.J. (1997) A General Model for the Origin of Allometric Scaling Laws in Biology. Science, 276, 122-126. https://doi.org/10.1126/science.276.5309.122

[40] Labra, F.A., Marquet, P.A. and Bozinovic, F. (2007) Scaling Metabolic Rate Fluctuations. Proceedings of the National Academy of Sciences of the United States of America, 104, 10900-10903. https://doi.org/10.1073/pnas.0704108104 
[41] West, G.B., Woodruff, W.H. and Brown, J.H. (2002) Allometric Scaling of Metabolic Rate from Molecules and Mitochondria to Cells and Mammals. Proceedings of the National Academy of Sciences of the United States of America, 99, 2473-2478. https://doi.org/10.1073/pnas.012579799

[42] Guiot, C., Delsanto, P.P.P., Carpinteri, A., Pugno, N., Mansury, Y. and Deisboeck, T.S. (2006) The Dynamic Evolution of the Power Exponent in a Universal Growth Model of Tumors. Journal of Theoretical Biology, 240, 459-463. https://doi.org/10.1016/j.jtbi.2005.10.006

[43] Puliafito, A., Primo, L. and Celani, A. (2017) Cell-Size Distribution in Epithelial Tissue Formation and Homeostasis. Journal of The Royal Society Interface, 14, Article ID: 20170032. https://doi.org/10.1098/rsif.2017.0032

[44] Egeblad, M., Nakasone, E.S. and Werb, Z. (2010) Tumors as Organs: Complex Tissues That Interface with the Entire Organism. Developmental Cell, 18, 884-901. https://doi.org/10.1016/j.devcel.2010.05.012

[45] Kallinowski, F., Schlenger, K.H., Runkel, S., Kloes, M., Stohrer, M., Okunieff, P. and Vaupel, P. (1989) Blood Flow, Metabolism, Cellular Microenvironment, and Growth Rate of Human Tumor Xenografts. Cancer Research, 49, 3759-3764.

[46] Guiot, C., Degiorgis, P.G, Delsanto, P.P., Gabriele, P. and Deisboeck, T.S. (2003) Does Tumor Growth Follow a "Universal Law"? Journal of Theoretical Biology, 225, 147-151. https://doi.org/10.1016/S0022-5193(03)00221-2

[47] Moatemed, F., Sahimi, M. and Naeim, F. (1998) Fractal Dimension of the Bone Marrow in Metastatic Lesions. Human Pathology, 29, 1299-1303. https://doi.org/10.1016/S0046-8177(98)90261-1

[48] West, G.B. and Brown, J.H. (2004) Life's Universal Scaling Laws. Physics Today, 57, 36-44. https://doi.org/10.1063/1.1809090

[49] West, G.B., Brown, J.H. and Enquist, B.J. (2001) A General Model for Ontogenic Groth. Nature, 413, 628-631. https://doi.org/10.1038/35098076

[50] Makarieva, A.M., Nefiodov, A.V. and Li, B.-L. (2020) Life's Energy and Information: Contrasting Evolution of Volume versus Surface-Specific Rates of Energy Consumption. Entropy, 22, Article No. 1025. https://doi.org/10.3390/e22091025

[51] Baish, J.W. and Jain, R.K. (2000) Fractals and Cancer. Cancer Research, 60, 3683-3688.

[52] Tubiana, M. (1989) Tumor Cell Proliferation Kinetics and Tumor Growth Rate. Acta Oncologica, 28, 113-121. https://doi.org/10.3109/02841868909111193

[53] Gazit, Y. (1996) Fractal Vasculature and Vascular Network Growth Modeling in Normal and Tumor Tissue. PhD Thesis, Massachusetts Institute of Technology, Cambridge, MA.

[54] Sabo, E., Boltenko, A., Sova, Y., Stein, A., Kleinhaus, S. and Resnick, M.B. (2001) Microscopic Analysis and Significance of Vascular Architectural Complexity in Renal Cell Carcinoma. Clinical Cancer Research, 7, 533-537.

[55] Herman, A.B., Savage, V.M. and West, G.B. (2011) A Quantitative Theory of Solid Tumor Growth, Metabolic Rate and Vascularization. PLoS ONE, 6, e22973. https://doi.org/10.1371/journal.pone.0022973

[56] Bauer, W. and Mackenzie, C.D. (1995) Cancer Detection via Determination of Fractal Cell Dimension. arXiv:patt-sol/9506003.

[57] Mandelbrot, B.B. and Wheeler, J.A. (1983) The Fractal Geometry of Nature. American Journal of Physics, 51, 286-287. https://doi.org/10.1119/1.13295

[58] Huang, W., Yen, R.T., McLaurine, M. and Bledsoe, G. (1996) Morphometry of the 
Human Pulmonary Vasculature. Journal of Applied Physiology, 81, 2123-2133. https://doi.org/10.1152/jappl.1996.81.5.2123

[59] Savage, Van M., Gillooly, J.F., Woodruff, W.H., West, G.B., Allen, A.P., Enquist, B.J. and Brown, J.H. (2014) The Predominance of Quarter-Power Scaling in Biology. Functional Ecology, 18, 257-282. https://doi.org/10.1111/j.0269-8463.2004.00856.x

[60] Szigeti, G.P., Szasz, O. and Hegyi, G. (2017) Connections between Warburg's and Szentgyorgyi's Approach about the Causes of Cancer. Journal of Neoplasm, 1, 1-13.

[61] Voet, D., Voet, J.G. and Pratt, C.W. (2006) Fundamentals of Biochemistry. 2nd Edition, John Wiley and Sons, Inc., Hoboken, 547, 556.

[62] Pamatmat, M.M. (2005) Measuring Aerobic and Anaerobic Metabolism of Benthic Infauna under Natural Conditions. Journal of Experimental Zoology, 228, 405-413. https://doi.org/10.1002/jez.1402280303

[63] Costello, L.C. and Franklin, R.B. (2006) Tumor Cell Metabolism: The Marriage of Molecular Genetics and Proteomics with Cellular Intermediary Metabolism; Proceed with Caution! Molecular Cancer, 5, Aticle No. 59. https://doi.org/10.1186/1476-4598-5-59

[64] Warburg, O. (1956) On the Origin of Cancer Cells. Science, 123, 309-314. https://doi.org/10.1126/science.123.3191.309

[65] Jeon, S.-M. and Hay, N. (2018) Expanding the Concepts of Cancer Metabolism. EXperimental \& Molecular Medicine, 50, 1-3. https://doi.org/10.1038/s12276-018-0070-9

[66] Ward, P.S. and Thompson, C.B. (2012) Metabolic Reprogramming: A Cancer Hallmark Even Warburg Did Not Anticipate. Cancer Cell, 21, 297-308. https://doi.org/10.1016/j.ccr.2012.02.014

[67] Hand, S.V. and Menze, M.A. (2008) Commentary Mitochondria in Energy-Limited States: Mechanisms That Blunt the Signaling of Cell Death. The Journal of Experimental Biology, 211, 1829-1840. https://doi.org/10.1242/jeb.000299

[68] Szasz, O., Vincze, G., Szigeti, G.P., Benyo, Z. and Szasz, A. (2018) An Allometric Approach of Tumor-Angiogenesis. Medical Hypotheses, 116, 74-78. https://doi.org/10.1016/j.mehy.2018.03.015

[69] Tannock, I.F. (1968) The Relation between Cell Proliferation and the Vascular System in a Transplanted Mouse Mammary Tumour. British Journal of Cancer, 22, 258-273. https://doi.org/10.1038/bjc.1968.34

[70] Milotti, E., Vyshemirsky, V., Sega, M. and Chignola, R. (2012) Interplay between Distribution of Live Cells and Growth Dynamics of Solid Tumours. Scientific Reports, 2, Article No. 990. https://doi.org/10.1038/srep00990

[71] Milotti, E., Vyshemirsky, V., Sega, M. and Chignola, R. (2013) Metabolic Scaling in Solid Tumours. Scientific Reports, 3, Article No. 1938. https://doi.org/10.1038/srep01938

[72] Painter, P.R. (2005) Allometric Scaling of the Maximum Metabolic Rate of Mammals: Oxygen Transport from the Lungs to the Heart Is a Limiting Step. Theoretical Biology and Medical Modelling, 2, Article No. 31. https://doi.org/10.1186/1742-4682-2-31

[73] Szasz, O. and Szigeti, Gy.P. (2020) Allometric Scaling by the Length of the Circulatory Network. Frontiers in Physiology Fractal and Network Physiology. (Under Review)

[74] Shim, E.B., Kim, Y.S. and Deisboeck, T.S. (2007) 2D FEM Tumor Angiogenesis Model 1 Analyzing the Dynamic Relationship between Tumor Growth and Angiogenesis in a Two Dimensional Finite Element Model. https://arxiv.org/ftp/q-bio/papers/0703/0703015.pdf 
[75] Landini, G. and Rippin, J.W. (1993) Fractal Dimensions of the Epithelial-Connective Tissue Interfaces in Premalignant and Malignant Epithelial Lesions of the Floor of the Mouth. Analytical and Quantitative Cytology and Histology, 15, 144-149.

[76] McDonald, M.D. and Choyke, P.L. (2003) Imaging of Angiogenesis from Microscope to Clinic. Nature Medicine, 9, 713-725.

https://doi.org/10.1038/nm0603-713

[77] Ichim, L. and Dobrescu, R. (2013) Characterization of Tumor Angiogenesis Using Fractal Measures. 19th International Conference on Control Systems and Computer Science, Bucharest, 29-31 May 2013, 345-349. https://doi.org/10.1109/CSCS.2013.18 https://www.researchgate.net/publication/261092279

[78] Jurczyszyn, K., Osiecka, B.J. and Ziołkowski, P. (2012) The Use of Fractal Dimension Analysis in Estimation of Blood Vessels Shape in Transplantable Mammary Adenocarcinoma inWistar Rats after Photodynamic Therapy Combined with Cysteine Protease Inhibitors. Computational and Mathematical Methods in Medicine, 2012, Article ID: 793291. https://doi.org/10.1155/2012/793291

[79] Avakian, A., Kalina, R.E., Sage, E.H., Rambhia, A.H., Elliott, K.E., Chuang, E.L., Clark, J.I., Hwang, J.N. and Parsons-Wingerter, P. (200) Fractal Analysis of Region-Based Vascular Change in the Normal and Non-Proliferative Diabetic retina. Current Eye Research, 24, 274-280.

https://doi.org/10.1076/ceyr.24.4.274.8411

[80] Parsons-Wingerter, P., Chandrasekharan, U.M., McKay, T.L., Radhakrishnan, K., DiCorleto, P.E., Albarran, B. and Farr, A.G. (2006) A VEGF165-Induced Phenotypic Switch from Increased Vessel Density to Increased Vessel Diameter and Increased Endothelial NOS Activity. Microvascular Research, 72, 91-100. https://doi.org/10.1016/j.mvr.2006.05.008

[81] Guidolin, D., Vacca, A., Nussdorfer, G.G. and Ribatti, D. (2004) A New Image Analysis Method Based on Topological and Fractal Parameters to Evaluate the Angiostatic Activity of Docetaxel by Using the Matrigel Assay in Vitro. Microvascular Research, 67, 117-124. https://doi.org/10.1016/j.mvr.2003.11.002

[82] Mancardi, D., Varetto, G., Bucci, E., Maniero, F. and Guiot, C. (2008) Fractal Parameters and Vascular Networks: Facts \& Artifacts. Theoretical Biology and Medical Modelling, 5, Article No. 12. https://doi.org/10.1186/1742-4682-5-12 